\title{
CARACTERIZAÇÃO DOS SISTEMAS DE GERAÇÃO ELÉTRICA DOS FPSOs EM OPERAÇÃO NO BRASIL
}

\author{
José Antônio Maciel Pereira ${ }^{1}$ \\ João Damasceno de Jesus ${ }^{2}$ \\ Eduardo Atem de Carvalho ${ }^{3}$
}

\begin{abstract}
Resumo: Com o advento da produção de petróleo e gás em áreas cada vez mais distantes da costa brasileira, o uso de FPSOs vem ganhando destaque devido a sua melhor adequação a esta demanda. Porém, necessitam de produção própria de energia elétrica para seus processos e, por conseguinte, possuem instaladas unidades autônomas de geração elétrica. Estas plantas de geração existentes são identificadas em função da máquina motriz e categorizadas em Tipo, Combinação, Potência Instalada, Quantidade e Fabricante. Verificou-se que três fabricantes estrangeiros dominam $80 \%$ do fornecimento de máquinas-motrizes nos FPSOs, com a predominância de turbinas a gás. Ao mesmo tempo, são apresentadas sugestões de esforços coordenados dos usuários para busca de melhores práticas que possam reduzir esta dependência. Um total de 1,8 GW de potência instalada em FPSOs são identificados, porém não são considerados no inventário nacional pela EPE, bem como estas plantas não possuem nenhum tipo de regulação específica das agências governamentais e existem oportunidades de melhor eficiência energéticaEste trabalho é um estudo caso e adota a data de corte de março de 2013.
\end{abstract}

Palavras-chave: FPSOs, geração elétrica, turbinas a gás.

Abstract: As far as oil production at sea bed on areas distant from brazilliancoast comes up, FPSOs application have been raised due to very good fitting for this requirement. Due this distance FPSOs need own power generation to get power available for all equipments on board. These power generation plants are identifying in light of power machinery and categorized by type of machine, installed power and manufacturer. Gas Turbines arise as the most used equipments with some room of efficiency three manufactures hold closer $80 \%$ of FPSOs. Some improvement suggestions are done to get users coordinated efforts aiming best practices and new technology development in order to get this dependency lower. FPSOs provide 1,7 GW of power, but this amount has not been considered within Brazil inventory published by EPE as well as away from any regulation by government agencies and there are much room for improvement energetic efficiency opportunities. This academic research is a case study and adopted thedeadline on March, 2013.

Keywords: FPSOs, Power generation, gas turbines.

\footnotetext{
${ }^{1}$ Faculdade Municipal Professor Miguel A. S. Santos - FEMASS, Macaé/RJ. E-mail: joseantonio.maciel@uol.com.br

${ }^{2}$ Instituto Federal Fluminense - IFF, Macaé/RJ. E-mail: joao.damasceno.jesus@gmail.com

${ }^{3}$ Universidade Estadual do Norte-Fluminense - UENF/Campos dos Goytacazes (RJ). E-mail: eatem@uenf.br
} 


\section{1 -INTRODUÇÃO}

Atualmente a grande parte da produção de petróleo e gás nacional ocorre em alto mar (offshore) e com as promessas pelas descobertas na área de pré-sal, esta operação tende a ser realizada em distâncias cada vez maiores em relação ao continente, o que eleva ainda mais os custos de uma possívelinstalação de novos oleodutos e gasodutos submarinos. Como solução para esta demanda natural as empresas do setor vêm se utilizando da aplicação de unidades marítimas flutuantes em alto mar. Estas instalações devem ter a flexibilidade necessária para que o óleo e o gás consigam ser escoados para um dado destino, desempenhando, assim, a sua função comercial. Nesta indústria offshore, as plataformas do tipo FPSO (Floating Production Storageand Offloading) apresentam grande destaque nos campos marítimos de petróleo. Segundo a Empresa de Pesquisa Energética (2014), em seu Plano Decenal de Energia, estima que no ano de 2021 haja a demanda para 90 FPSOsem operação ao longo da costa brasileira

Os Sistemas de Geração Elétrica dos FPSOs configuram-se como unidades termelétricas flutuantes em alto mar, tendogeração de energia própria e independente para que seja assegurada a alimentação elétrica para os sistemas de energia para todos os processos de produção da unidade. Ao mesmo tempo, existindoum sistema de energia de emergência que supra as necessidades essenciais para o caso de interrupção desta energia.

Tavares (2010) divide os sistemas de Geração Elétrica, de um modo geral, em cargas elétricas distribuídas em ditas Normais e Essenciais, Normais são alimentadas exclusivamente pelo Sistema de Geração Principal e devem permanecer desernegizadas, enquanto a Geração Principal estiver desligada. Por outro lado, as Essenciais são normalmente alimentadas pela Geração Principal e que passam a ser alimentadas por um Gerador de Emergência no caso da falha da Geração Principal. Neste trabalho, não serão considerados os Geradores de Emergência por não fazerem parte diretamente da operação do FPSO e, deste modo, Sistemas de Geração Elétrica e Geração Principal terão o mesmo significado.

Os sistemas de Geração Elétrica são compostos por uma máquina (geralmente térmica) que é acoplada a um gerador elétrico. Normalmente, são instalados em módulos ou também se utilizam da antiga planta de Geração Elétrica do navio, que foi convertido para FPSO. Em geral, estas máquinas primárias também podem ser acopladas em Compressores Rotativos, Alternativos ou Bombas de Grande porte para deslocamento de fluidos.

Diversos trabalhos acadêmicos sobre FPSOs já são encontrados, com foco, principalmente, na produção de óleo e gás, como, por exemplo, Meneses (2011) e Oliveira (2013). Porém, não são encontrados trabalhos publicados que identifiquem e caracterizem os sistemas de geração elétrica destas unidades.

O objetivo desta pesquisa é contribuir para o desenvolvimento do tema, identificando e caracterizando os Sistemas de Geração de Energia Elétrica dos 34 FPSOs instalados e em operação no Brasil, até o mês de março do ano de 2013, nas Bacias de Santos, Campos, Espírito Santo e Sergipe-Alagoas. Deste modo, pretende-se apresentar, através de uma pesquisa qualitativa e descritiva, estes importantes sistemas tão vitais para a completa operação das Unidades. Neste artigo, por limitação de escopo, não serão abordados os geradores elétricos, que são as máquinas que produzem energia elétrica, e sim, as máquinas motrizes que os impulsionam.

\section{2 - DESENVOLVIMENTO}

\subsection{FPSO}

É uma unidade industrial flutuante, construída ou convertida por uma reforma e adaptação grandes petroleiros tipo VLCC (Very Large Crude Carrier) e ULCC (Ultra Large Crude Carrier), em geral, fabricados na 
década de 1970. De acordo com a Consultoria 2b1stconsulting (2013), aconversão é a prática mais utilizada no mercado, pois possui um foco em redução de custos, entrega mais rápida pelo estaleiro e melhor gestão do ativo.Kawasaki (2010) mostra que asunidades são utilizadas para desenvolvimento de operações de petróleo e gás em lâminas de água intermediárias, profundas e ultraprofundas e, segundo Amorim (2010), são fixadas no fundo do mar através de sistemas de ancoragem e tendo como idéia central a garantia de uma grande capacidade de armazenamento. $\mathrm{O}$ posicionamento dessas unidades ocorre em campos muito afastados da costa, onde a instalação de linhas de dutos torna-se um fator de alto custo, conforme Grove (2005), podendo operar por até 25 anos sem necessidade de manutenção em estaleiros (docagem). Devem impactar o mínimo possível o meio ambiente, bem como a um custo que atenda o critério econômico do projeto para que seja entregue $o$ valor do investimento projetado (OLF, 2011). Apresentam um menor custo global e grande flexibilidade de ser instalado em qualquer campo offshore, que se traduz em uma maior eficiência, com apenas adaptações de engenharia, sendo utilizados para produção, processamento do petróleo e gás, armazenamento e transferência (offloading) desta produção de petróleo para navios aliviadores.

\subsection{SISTEMAS DE GERAÇÃO ELÉTRICA EM UNIDADES FLUTUANTES}

O Sistema de Geração Elétrica possui uma função crítica em um FPSO (OLF, 2011), que, no Brasil, deve atender diversas normas de construção, projeto, segurança, estatutárias e as pertinentes à bandeira existente na embarcação. Dentre estas, podemos citar as Normas IEC (International

Electrotechnical Comission) 60034 (ISO 8528-3) e as determinadas pela Sociedade Classificadora. Este conjunto prescreve as condições de projeto e aplicação em unidades fixas e móveis na indústria marítima. Tavares (2010) estabelece que o Sistema de Geração Elétrica é equipado com dispositivos, que em condições anormais de operação emitem alarmes, mantendo-o em funcionamento ou $\mathrm{o}$ paralisando como proteção contra os danos.

Normalmente, o Sistema de Geração é composto por conjuntos de máquinas motrizes ou primárias que acionam geradores elétricos que, por sua vez, transformam a energia mecânica de rotação das máquinas primárias em energia elétrica. Meneses (2011) estabelece que, em regra geral, a escolha da máquina motriz é dependente do tipo de gerador que será utilizado no sistema. Sua capacidadeé projetada para operar continuamente para atender o consumo de cargas para operação, bem como os casos de pico previstos para a unidade, garantindo assim uma capacidade redundante de segurança para atender as demandas de alta disponibilidade da unidade (DNV, 2012).

Tolmasquim (2005) estabelece máquinas motrizes que são utilizadas para impulsionar um gerador elétrico: motores de combustão interna, turbinas a gás, motores de combustão externa, turbinas a vapor. Contudo, já existem estudos para a utilização de energia eólica para FPSOs e uso de LNG, que podem ter exemplos verificados em Oliveira (2013).

Em caso de máquinas motrizes à combustão, o uso de gás natural ou óleo diesel fornecem maior confiabilidade e flexibilidade na operação (OLF, 2011). Em geral, devido à sua facilidade de obtenção e abundância no próprio processo de exploração do poço petrolífero na operação normal, o gás natural é a primeira opção, ficando o óleo diesel como backup em caso de falta do anterior. Outra característica é o reaproveitamento do calor existente nos gases de combustão para o aquecimento de fluidos auxiliares na Planta de Utilidades através de Caldeiras de Recuperação de Calor (WHRU: Waste Heat Recovery Units). 


\section{3 -METODOLOGIA}

Buscando estabelecer a descrição em profundidade das Máquinas Motrizes dos Sistemas de Geração Elétrica dos FPSOs no Brasil, optou-se pelo estudo de $100 \%$ da amostra estudada, ou seja, toda a população. A partir do trabalho de campo, os dados da pesquisa foram coletados no período de maio a agosto de 2013 a partir de fontes múltiplas de evidências, ou seja, utilizou-se os meios de pesquisa documental da internet, comunicação através de e-mail para as empresas, acesso através de mídia de relacionamento profissional (Linked in) e contato telefônico.

Para a categorização dos FPSOs, foi utilizada a listagem disponível na literatura técnica-comercial, quando foram identificadas 34 unidades operando na costa nacional, divididas pelas Bacias de Campos, Santos, Espírito Santo e Sergipe-Alagoas (RBEPG, 2013). A linha de corte da pesquisa foi definida com a data de 31 demarço de 2013.

As Plantas de Geração Elétrica dos FPSOs são categorizadas quanto a algumas características e refletindo esta composiçãoquanto à totalidade da Frota, obedecendo aos seguintes critérios:

a) Quanto ao Tipo de Máquina Motriz, adaptando a tipologia apresentada por Tomalsquim (2005): motores de combustão interna (MCI), turbinas a gás (TG), motores de combustão externa (MCE) e turbinas a vapor (TV).

b) Quanto à Combinação de Máquinas Motrizes: simples (somente um tipo de máquina motriz), mista (somente dois tipos de máquinas motrizes) e múltipla (dois ou mais máquinas motrizes).

c) Quanto à Potência Instalada a partir das Máquinas Motrizes que compõem a Planta de Geração Elétrica: baixa (0 a 30MW), média (30 a $50 \mathrm{MW}$ ) e alta (acima de $50 \mathrm{MW}$ ).

d) Quanto ao Número de Máquinas Motrizes instaladas. e) Quanto aos Fabricantes das Máquinas Motrizes instaladas.

\section{4 - RESULTADOS ENCONTRADOS}

Na compreensão do fenômeno, que pode estar ligado ao estudo de caso e com a proposta de obter explicações sobre a realidade existente entre a geração de energia em FPSOs e as máquinas motrizes que impulsionam os seus geradores elétricos, são apresentadas as evidências empíricas que foram levantadas a partir de fontes variadas do trabalho de campo.

A Tabela 1 mostra os FPSOs em operação em diversos campos de produção existentes ao longo da costa do Brasil conforme a Revista Brasil Energia do mês de março de 2013. 
Tabela 1 - Unidades FPSOs em operação no Brasil em março de 2013 (Revista Brasil Energia Petróleo e Gás 2013)

\begin{tabular}{|c|c|c|c|}
\hline $\begin{array}{c}\text { Unidade } \\
\text { FPSO }\end{array}$ & Proprietário & $\begin{array}{l}\text { Unidade } \\
\text { FPSO }\end{array}$ & $\begin{array}{c}\text { Proprietá } \\
\text { rio }\end{array}$ \\
\hline Brasil & SBM & OSX - 1 & osx \\
\hline Capixaba & SBM & P-31 & Petrobras \\
\hline $\begin{array}{c}\text { Cidade Angra } \\
\text { dos Reis }\end{array}$ & Modec & P-33 & Petrobras \\
\hline $\begin{array}{l}\text { Cidade de } \\
\text { Anchieta }\end{array}$ & SBM & P-34 & Petrobras \\
\hline $\begin{array}{l}\text { Cidade de } \\
\text { Itajaí }\end{array}$ & ODN/Teekay & P-35 & Petrobras \\
\hline $\begin{array}{c}\text { Cidade de } \\
\text { Niterói }\end{array}$ & Modec & P-37 & Petrobras \\
\hline $\begin{array}{c}\text { Cidade de } \\
\text { Santos }\end{array}$ & Modec & P-43 & Petrobras \\
\hline $\begin{array}{l}\text { Cidade de } \\
\text { São Paulo }\end{array}$ & $\begin{array}{l}\text { Schahin/ } \\
\text { Modec }\end{array}$ & P-47 & Petrobras \\
\hline $\begin{array}{l}\text { Cidade Rio } \\
\text { das Ostras }\end{array}$ & Petrojarl & P-48 & Petrobras \\
\hline $\begin{array}{c}\text { Cidade São } \\
\text { Mateus }\end{array}$ & BW Offshore & P-50 & Petrobras \\
\hline $\begin{array}{c}\text { Cidade São } \\
\text { Vicente }\end{array}$ & BW Offshore & P-53 & Petrobras \\
\hline $\begin{array}{c}\text { Cidade de } \\
\text { Vitória }\end{array}$ & Saipem & P-54 & Petrobras \\
\hline $\begin{array}{l}\text { Dynamic } \\
\text { Producer }\end{array}$ & Petroserv & P-57 & Petrobras \\
\hline $\begin{array}{c}\text { Espirito } \\
\text { Santo } \\
\end{array}$ & SBM & Peregrino & Maersk \\
\hline Fluminense & Modec & Piranema & Teekay \\
\hline Frade & Chevron & Polvo & $\begin{array}{c}\text { BW } \\
\text { Offshore }\end{array}$ \\
\hline Marlim Sul & SBM & $\begin{array}{c}\text { Rio de } \\
\text { Janeiro }\end{array}$ & Modec \\
\hline
\end{tabular}

Tabela 2: Identificação das Plantas de Geração Elétrica de Unidades FPSOs em operação no Brasil em março de 2013 em relação à Potência Instalada (MW).

\begin{tabular}{|c|c|c|c|c|c|}
\hline Unidade FPSO & $\begin{array}{c}\text { Potência Instalada } \\
\text { (MW) }\end{array}$ & Unidade FPSO & $\begin{array}{c}\text { Potência } \\
\text { Instalada (MW) }\end{array}$ & Unidade FPSO & $\begin{array}{c}\text { Potência } \\
\text { Instalada (MW) } \\
\end{array}$ \\
\hline Brasil & 48 & $\begin{array}{l}\text { Dynamic } \\
\text { Producer }\end{array}$ & 22 & P-43 & 92 \\
\hline Capixaba & 67 & Espirito Santo & 64 & P-47 & 7,7 \\
\hline $\begin{array}{c}\text { Cidade Angra } \\
\text { dos Reis }\end{array}$ & 92 & Fluminense & 32 & P-48 & 92 \\
\hline $\begin{array}{c}\text { Cidade de } \\
\text { Anchieta } \\
\end{array}$ & 16 & Frade & 92 & P-50 & 92 \\
\hline Cidade de Itajaí & 36 & Marlim Sul & 72 & P-53 & 92 \\
\hline $\begin{array}{c}\text { Cidade de } \\
\text { Niterói }\end{array}$ & 69 & OSX-1 & 42 & P-54 & 92 \\
\hline $\begin{array}{c}\text { Cidade de } \\
\text { Santos } \\
\end{array}$ & 92 & P-31 & 6 & P-57 & 92 \\
\hline $\begin{array}{c}\text { Cidade de São } \\
\text { Paulo }\end{array}$ & 69 & P-33 & 10 & Peregrino & 72 \\
\hline $\begin{array}{c}\text { Cidade Rio das } \\
\text { Ostras }\end{array}$ & 7 & P-34 & 7 & Piranema & 8 \\
\hline $\begin{array}{c}\text { Cidade São } \\
\text { Mateus }\end{array}$ & 30 & P-35 & 20 & Polvo & 38 \\
\hline $\begin{array}{c}\text { Cidade São } \\
\text { Vicente }\end{array}$ & 4 & P-37 & 92 & Rio de Janeiro & 42 \\
\hline $\begin{array}{c}\text { Cidade de } \\
\text { Vitória }\end{array}$ & 53 & * & * & * & * \\
\hline
\end{tabular}


Tabela 3 - Identificação das Plantas de Geração Elétrica de Unidades FPSOs em operação no Brasil em março de 2013 em relação à Máquina Motriz e aos Fabricantes.

\begin{tabular}{|c|c|c|c|c|c|}
\hline Unidade & Máquina Motriz & Fabricante & Unidade & Máquina Motriz & Fabricante \\
\hline \multirow{2}{*}{ Brasil } & Turbinas a Gás & Siemens & Frade & Turbina a Gás & General Electric \\
\hline & Turbina a Vapor & Não Identificado & Marlim Sul & Turbina a Vapor & Dresser-Rand \\
\hline \multirow{3}{*}{ Capixaba } & Turbina a Gás & Sollar & \multirow{2}{*}{ OSX - 1} & Turbina a Gás & Solar \\
\hline & Turbina a Vapor & Não Identificado & & $\mathrm{MCl}$ & Hyundai \\
\hline & $\mathrm{MCl}$ & Não Identificado & P-31 & Turbina a Vapor & Siemens \\
\hline $\begin{array}{l}\text { Cidade Angra } \\
\text { dos Reis }\end{array}$ & Turbina a Gás & General Electric & \multirow{2}{*}{ P-33 } & Turbina a Vapor & Ishikawajima \\
\hline $\begin{array}{l}\text { Cidade de } \\
\text { Anchieta }\end{array}$ & Turbina a Gás & ABB/Alstom & & $\mathrm{MCl}$ & Wartsila \\
\hline \multirow{2}{*}{ Cidade de Itajaí } & Turbina a Gás & Siemens & P-34 & $\mathrm{MCl}$ & Não Identificado \\
\hline & $\mathrm{MCl}$ & Hyundai & P-35 & Turbina a Gás & EGT/Alstom \\
\hline Cidade de Niterói & Turbina a Gás & General Electric & P-37 & Turbina a Gás & General Electric \\
\hline Cidade de Santos & Turbina a Gás & General Electric & $P-43$ & Turbina a Gás & Rolls-Royce \\
\hline $\begin{array}{c}\text { Cidade de São } \\
\text { Paulo }\end{array}$ & Turbina a Gás & General Electric & P-47 & $\mathrm{MCl}$ & Wartsila \\
\hline \multirow{2}{*}{$\begin{array}{l}\text { Cidade Rio das } \\
\text { Ostras }\end{array}$} & $\mathrm{MCl}$ & MAK & P-48 & Turbina a Gás & Rolls-Royce \\
\hline & $\mathrm{MCl}$ & Wartsila & P-50 & Turbina a Gás & General Electric \\
\hline $\begin{array}{c}\text { Cidade São } \\
\text { Mateus }\end{array}$ & Turbina a Vapor & Shinko & P-53 & Turbina a Gás & Rolls-Royce \\
\hline \multirow{2}{*}{$\begin{array}{l}\text { Cidade São } \\
\text { Vicente }\end{array}$} & Turbina a Vapor & Shinko & P-54 & Turbina a Gás & General Electric \\
\hline & $\mathrm{MCl}$ & Caterpillar & P-57 & Turbina a Gás & General Electric \\
\hline \multirow{2}{*}{ Cidade de Vitória } & Turbina a Gás & Solar & Peregrino & Turbinas a Vapor & Dresser-Rand \\
\hline & Turbina a Vapor & Dresser-Rand & Piranema & Turbina a Gás & OPRA \\
\hline $\begin{array}{l}\text { Dynamic } \\
\text { Producer }\end{array}$ & $\mathrm{MCl}$ & Wartsila & \multirow{2}{*}{ Polvo } & Turbina a Vapor & Não Identificado \\
\hline \multirow{2}{*}{ Espirito Santo } & Turbina a Gás & Solar & & $\mathrm{MCl}$ & Daihatsu \\
\hline & Turbina a Vapor & Dresser-Rand & Rio de Janeiro & Turbina a Gás & Solar \\
\hline Fluminense & Turbina a Gás & Solar & & & \\
\hline
\end{tabular}

As Tabelas 02 e 03 já apresentam a identificação das Plantas frente à Potência Instalada e Fabricantes das Máquinas Motrizes respectivamente.

Deste universo destas 34 Unidades de Produção FPSOs produzem um total de 3,489 milhões de barris por dia, em sua grande totalidade a serviço (próprios ou afretados) da Petrobrás. Deste modo, a Petrobras se configura como principal player do mercado.

A primeira unidade foi instalada no ano de 1979 e até o ano de 2015, dez novos FPSOs entrarão em operação (RBEPG, 2013).

\section{5 - CATEGORIZAÇÃo.}

A categorização das Plantas de Geração Elétrica, em referência aos critérios vistos na Metodologia, é apresentada nas Tabelas 4, 5, 6, 7, 8 e 9.
Tabela 4: Categorização das Plantas de Geração Elétrica de Unidades FPSOs em operação no Brasil em março de 2013 em relação às máquinas motrizes

\begin{tabular}{|c|c|c|}
\hline Plantas de GeraçãoElétrica & FPSOs & $\begin{array}{c}\% \text { da } \\
\text { Frota }\end{array}$ \\
\hline SomenteTurbinas a Gás & 17 & $50,0 \%$ \\
\hline SomenteTurbinas a Vapor & 4 & $11,8 \%$ \\
\hline SomenteMCl & 4 & $11,8 \%$ \\
\hline $\begin{array}{c}\text { Turbinas a Gás + Turbinas a } \\
\text { Vapor }\end{array}$ & 3 & $8,8 \%$ \\
\hline Turbinas a Vapor + MCl & 3 & $8,8 \%$ \\
\hline $\begin{array}{c}\text { Turbina a Gás + MCl } \\
\text { Turbina a Gás + Turbina a } \\
\text { Vapor + MCl }\end{array}$ & 1 & $5,9 \%$ \\
\hline Total & 34 & $100 \%$ \\
\hline
\end{tabular}


Tabela 5 - Categorização das Plantas de Geração Elétrica de Unidades FPSOs em operação no Brasil em março de 2013 quanto à combinação de máquinas motrizes.

\begin{tabular}{|c|c|c|}
\hline Plantas de Geração Elétrica & FPSO & $\begin{array}{c}\% \text { da } \\
\text { Frota }\end{array}$ \\
\hline $\begin{array}{c}\text { Simples (Somente um Tipo de } \\
\text { Máquina Motriz) }\end{array}$ & 25 & $73,6 \%$ \\
\hline $\begin{array}{c}\text { Mista (Somente Dois Tipos de } \\
\text { Máquinas Motrizes) }\end{array}$ & 8 & $23,5 \%$ \\
\hline $\begin{array}{c}\text { Múltipla (Dois ou mais Tipos de } \\
\text { Máquinas Motrizes) }\end{array}$ & 1 & $2,9 \%$ \\
\hline
\end{tabular}

Tabela 6 - Categorização das Plantas de Geração Elétrica de Unidades FPSOs em operação no Brasil em março de 2013 em relação à Potência Instalada.

\begin{tabular}{|c|c|c|}
\hline Potêncialnstalada & FPSO & \% da Frota \\
\hline Alta (acima de $50 \mathrm{MW})$ & 17 & $50 \%$ \\
\hline Baixa (0 a 30MW) & 11 & $32 \%$ \\
\hline Média (30 a $50 \mathrm{MW})$ & 6 & $18 \%$ \\
\hline
\end{tabular}

Tabela 7 - Categorização das Plantas de Geração Elétrica de Unidades FPSOs em operação no Brasil em março de $2013 \mathrm{em}$ relação às Máquinas Motrizes Instaladas.

\begin{tabular}{|c|c|}
\hline MáquinasMotrizesInstaladas & Unidades \\
\hline Turbinas a Gás & 83 \\
\hline $\mathrm{MCl}$ & 32 \\
\hline Turbinas a Vapor & 26 \\
\hline
\end{tabular}

Tabela 8 - Categorização das Plantas de Geração Elétrica de Unidades FPSOs em operação no Brasil em março de 2013 em relação ao Fabricante de Turbinas a Gás Instaladas.

\begin{tabular}{|c|c|c|}
\hline \multicolumn{3}{|c|}{ Composição por Fabricante - Turbinas a Gás } \\
\hline Fabricante & Unidades & FPSO Fleet $\%$ \\
\hline General Electric & 34 & $41,0 \%$ \\
\hline Solar & 19 & $22,9 \%$ \\
\hline Rolls-Royce & 12 & $14,5 \%$ \\
\hline Alstom (ABB+EGT) & 8 & $9,6 \%$ \\
\hline Siemens & 4 & $8,4 \%$ \\
\hline OPRA & 3 & $3,6 \%$ \\
\hline Total & 83 & $100,0 \%$ \\
\hline
\end{tabular}

Tabela 9 - Categorização das Plantas de Geração Elétrica de Unidades FPSOs em operação no Brasil em março de 2013 em relação ao Fabricante de Turbinas a Vapor Instaladas.

\begin{tabular}{|c|c|c|}
\hline \multicolumn{3}{|c|}{ Composição por Fabricante - Turbinas a Vapor } \\
\hline Fabricante & Unidades & FPSO Fleet \% \\
\hline Dresser-Rand & 9 & $34,6 \%$ \\
\hline Nãoldentificado & 8 & $30,8 \%$ \\
\hline Shinko & 5 & $19,2 \%$ \\
\hline Siemens & 2 & $7,7 \%$ \\
\hline Ishikawajima & 2 & $7,7 \%$ \\
\hline Total & 26 & $100,0 \%$ \\
\hline
\end{tabular}

Tabela 10 - Categorização das Plantas de Geração Elétrica de Unidades FPSOs em operação no Brasil em março de 2013 em relação ao Fabricante de Motores de Combustão Interna (MCI) Instalados.

\begin{tabular}{|c|c|c|}
\hline \multicolumn{3}{|c|}{ Composição por Fabricante - Motores Combustão } \\
Interna
\end{tabular}

\section{6 - ANÁLISE}

Verifica-se que o total de potência atualmente instalada é nos FPSOs é da ordem de $1.758 \mathrm{MW}$, sendo suficiente para alimentar uma cidade de cerca de 11,5 milhões de habitantes. Ao mesmo tempo, quase equivalendo à soma da potência instalada das duas maiores Usinas Termelétricas do país: UTE Governador Leonel Brizola (1058 MW) e UTE Mário Lago (822 MW), ambas no Rio de Janeiro. Aliado a isto, novos FPSOs são previstos no Plano Decenal de Expansão de Energia - Relatório Final do PDE 2021 (EPE, 2013).

Estas plantas de energia elétrica não possuem regulação específica para a operação. Apesar de não estarem em um 
ambiente de fornecimento de energia elétrica regulada, poderiam ser considerados como auto-produtores, passando daí a se nortearem pela regras vigentes, principalmente quanto a indicadores sustentáveis, tais como emissões por ação do homem e eficiência energética no uso do gás natural. Assim, deveriam receber um tratamento diferente dos Órgãos Reguladores, tais como a ANP (Agência Nacional de Petróleo) e ANEEL (Agência Nacional de Energia Elétrica).

Esta capacidade instalada nesta amostrado setor offshore, que também engloba plataformas outras que não FPSOs, não é computada no âmbito do Balanço Energético Nacional emitido pela EPE (Empresa de Pesquisa Energética), pois os setores abarcados na análise não mencionam claramente esta área em estudo, tanto na oferta, bem como demanda. Há apenas menção para as Indústrias de Transformação de energia, ou seja, onde a energia que entra (primária e/ou secundária) transforma-se em uma ou mais formas de energia secundária com suas correspondentes perdas na transformação (BEN, 2013).

Ao se apresentarem os fabricantes demonstra-se, o domínio do fabricante norte-americano General Electric no fornecimento de turbinas a gás, do fabricante finlandês Wartsila na entrega de motores de combustão interna e a outra americana Dresser-Rand na instalação de turbinas a vapor. Em relação ao aparecimento da General Electric como a maior fornecedora de turbinas para os FPSOs em operação, corrobora que o Brasil é o segundo mercado do mundo em turbinas a gás após o EUA (GE, 2011). Por outro lado, ratifica o uso de turbinas aero-derivativas de menor porte no meio offshore, tais como a GE LM 2500 (OLF, 2011) e a Rolls-Royce RB211 em sua grande maioria pela boa aplicação, confiabilidade e flexibilidade. Experiências não bem sucedidas com turbinas de maior potência no meio offshore, como as utilizadas em FPSOs na Noruega, são apontadas como um possível problema (OLF, 2011). Note-se que, conforme as evidências, a General Electric, Solar e Rolls-Royce possuem cerca de $80 \%$ de turbinas a gás instaladas, o que pode sugerir que estas três empresas dominariam e concentram o mercado local, garantindo um maior poder de barganha ao seu favor em negociações com os seus clientes. Ao mesmo tempo, o mercado torna-se cada vez mais concentrado através do movimento da aquisição pelo grupo alemão Siemens das áreas de produção de turbinas a gás e vapor das empresas Rolls-Royce (SIEMENS, 2014-1) e Dresser-Rand (SIEMENS, 2014-2). Destaque-se que a empresa francesa Alstom já é proprietária das marcas $\mathrm{ABB}$ e EGT.

Para melhor suporte, atendendo às exigências dos clientes para serviços e produtos nacionais incluindo reparos de motores e componentes, estas três empresas já possuem centros de serviço e de tecnologia no Brasil, concentrados na região sudeste do país. Esta regionalização área pode ser justificada pela instalação atual da maioria dos FPSOs nas Bacias de Campos (RJ) e de Santos (SP), bem como as demais plantas de geração em outros tipos de plataformas offshore. Outro fator de contribuição seria que o maior cliente destas empresas, a Petrobras, ter o seu centro de pesquisas, na Ilha do Fundão (RJ) e seus Centros de Serviços para Turbinas na cidade de Macaé (Parque de Tubos e UTE Mário Lago), ambas no Estado do Rio de Janeiro. A grande vantagem de um Centro de Serviços é para reforçar a capacidade de atendimento aos clientes no país, reduzindo o tempo de serviço pela metade, pois elimina boa parte da logística necessária para o envio da máquina ao exterior e os seus pesados custos, já que realiza serviços mais específicos nos equipamentos no país. Estes Centros de Serviços favorecem investimento contínuo em pesquisa e desenvolvimento de tecnologias em desempenho, flexibilidade operacional, buscando agregar cada vez mais valor ao negócio do cliente. (GE, 2011). 
Também como estratégia de mercado os fabricantes, de um modo geral, buscam desenvolver a área de pósvenda (afterselling) com a criação de departamentos internos ou cargos específicos para certos cliente, ditos "Gerentes de Conta", com o escopo de atendimento cada vez mais customizado para grandes clientes com o foco de venda de contratos de fornecimento de peças, reparos, operação e manutenção. $\mathrm{O}$ poder de barganha do fabricante é aumentado no momento em que alguns usuários das máquinas motrizes preferem utilizá-los diretamente a buscarem novas opções. Isto gera certo tradeoff no mercado e poderia ser explicado pela necessidade do usuário reduzir a sua incerteza do negócio ao buscar a confiabilidade prometida pelo fabricante. Este tipo de decisão impede $o$ desenvolvimento de novos participantes deste mercado, o que tende a concentrar ainda mais a tecnologia nos fabricantes estrangeiros.

\section{7 -CONCLUSÃo}

Este trabalho acadêmico apresentou a caracterização das Plantas de Geração de Energia Elétrica das Unidades FPSO a partir da identificação das máquinas motrizes que impelem os geradores elétricos. Esta caracterização e consequente composição frente à frota em operação existente deu-se com foco em Tipo, Combinação, Potência Instalada, Quantidade e Fabricante.

Com cerca de 1,8 GW de capacidade instalada em FPSOs figura como uma amostra significativa do setor offshore, acarretando que esta importante área da economia nacional, seja computada explicitamente no Balanço Energético Nacional (BEN) ou como pertencentes ao Parque Gerador Nacional pela ANEEL, pois faz parte da configuração das variáveis físicas próprias do setor energético e, deste modo, proporciona um panorama mais claro do setor.Além disto, existiria espaço para geração de um entendimento de como a ANEEL ou ANP, apesar de ser uma área não regulada, poderiam se envolver nesta área offshore, talvez com foco na sustentabilidade, como, por exemplo, controle de emissões atmosféricas, em plena sintonia com os desafios da sociedade de hoje.

Estas máquinas motrizes trabalham em ciclo aberto, onde o calor dos gases de descarga é recuperado para uso de vapor saturado para aquecimento em geral. Como a capacidade de elevação desta temperatura de vapor para níveis supersaturado é restrita, onde justificaria a aplicação de um ciclo combinado, com a instalação de uma turbina a vapor, estudos para a utilização de tecnologias tais como a queima destes gases de descarga ou regeneração poderiam ser avaliadas.

Esta maciça presença de turbinas a gás na composição destas Plantas em alto mar tende a ser uma amostra significativa da predominância destes equipamentos na indústria offshore em suas diversas plataformas.Daí, esforços devem ser envidados por empresas nacionais, usuários, universidades e poder público para garantir que a inovação seja buscada com a revitalização, por exemplo, da Rede de Turbinas a Gás (RTG) ou estímulos para a cadeia fornecedora de peças, serviços e consultorias nesta área.

A tecnologia de máquinas motrizes demonstra-se madura e garante, de certo modo, a dependência de peças, componentes e serviços de empresas estrangeiras. Os fabricantes estrangeiros dominam o fornecimento de máquinas motrizes para os FPSOs, pois o Brasil ainda não possui tecnologia própria para que consiga uma participação competitiva. Estes buscam, por sua vez, em sua estratégiade mercado promovema instalação de unidades de serviço e centros de tecnologia na Região Sudeste para atendimento à demanda dos clientes.

Apesar da figura do conteúdo local nos processos de construção de novas unidades, ainda é incerto se estas tecnologias serão repassadas aos usuários ou em que nível estes terão acesso. O que se hoje verifica é a dependência destes fabricantes, a partir da existência de contratos de operação e manutenção 
destes equipamentos. A superação desta dependência passaria pelo estágio atual do país em buscar trazer os fabricantes para a produção de novas tecnologias no país de modo compartilhado. Isto, de certo modo, é buscado na decisão de receber centros de pesquisas destes fabricantes, como, por exemplo, os Centros de Tecnologia da General Electric e da Rolls-Royce, ambos instalados no Rio de Janeiro.

Em relação aos usuários, recomenda-se o desenvolvimento de uma estratégia de abordagem de ações coordenadas do mercado frente aos fornecedores de turbinas a gás, na busca de melhores práticas de atendimento às demandas no offshore brasileiro, tais como um Fórum de Usuários, buscando um maior envolvimento no Estado Arte desta tecnologia, na busca de lições aprendidas para serem aplicadas em novas unidades a serem construídas ou convertidas no Brasil, bem como aprendizado em assuntos ligados a sua operação. Serve como referência o Grupo de Manutenção de Máquinas Térmicas (GTMT) da Associação Brasileira de Empresas de Geração Elétrica (ABRAGE).

Uma restrição a ser levada em conta neste artigo é a quantidade expressiva de $30,8 \%$ das turbinas a vapor não tiveram os seus fabricantes identificados pela pesquisa, acarretando uma incerteza na definição do maior fabricante.

Descrevendo as máquinas motrizes do Sistema de Geração Elétrica dos FPSOs no Brasil este artigo torna-se relevante com o seu propósito e inovador ao contribuir com informações importantes à indústria e à Academia.

O mercado de FPSOs no país é bastante promissor, tendendo hoje a crescer e novas unidades serão instaladas, como, por exemplo, a série de "replicantes" da Petrobras. Deste modo, convidando que este trabalho seja revisitado em breve futuro.

\section{Referências}

Associação Brasileira de Normas Técnicas:http://www.abntcatalogo.com.br /norma.aspx? $\mathrm{ID}=196027$. Acesso em $15 / 08 / 2013$

2b1st consulting:Disponível em http://www.2b1stconsulting.com/convert ed-fpso/Acesso em 15/08/2013.

AMORIM, T. O. Plataformas Offshore: uma Breve Análise desde a Construção ao Descomissionamento. (MonografiadeGraduação de Curso de Tecnologia Naval). Centro UniversitárioEstadual da Zona Oeste, Rio de Janeiro,2010.

Empresa de Pesquisa Energética Balanço Energético Nacional - Ano Base 2012. Relatório Final, 2013.

Empresa de Pesquisa Energética - EPE Relatório do Plano Decenal de Expansão de Energia 2021, 2014.

COCHRAN, I. Business opportunities in FPSOs Untangling the FPSO market:A Tanker Operator. July. Disponível em http://ea45bb970b5c70169c610cd083ee9 2972834b7bec0d968bf8995.r81.cf1.rackc dn.com/FPSOreportsample.pdf.

(2013)

Acesso em 19/07/2013.

DNV (2012) Innovation Project at oil and gasindustryOpera.Disponível em http://www.dnvusa.com/Binaries/Opera\% 20brochure tcm153-527124.pdf. Acesso em 25/06/2013

Featuresof

FPSO

Construction.Disponível em http://www.toyoeng.co.jp/en/product_line/development/fp so/index.html. Acesso em 22/06/2013.

$\begin{array}{lcr}\text { GENERAL } & \text { ELETRIC } & \text { PRESS } \\ \text { RELEASE. } & \text { Disponível } & \text { em }\end{array}$ http://geimprensabrasil.hospedagemdesite s.ws/wp-content/uploads/2011/11/19-deoutubro-de-2011-GE-inaugura-primeiroCentro-de-Servi\%C3\%A7os-paraturbinas-aeroderivadas-da-

Am\%C3\%A9rica-Latina.pdf. Acesso em 25/07/2013. 
GROVE, M.A., Sistema de Posicionamento Híbrido para FPSOs. (Dissertação de Mestrado em Engenharia Oceânica. Rio de Janeiro: Programa de Pós-Graduação de Engenharia), Universidade Federal do Rio de Janeiro 2005.

KAWASAKI, P. Y. Análise de Linhas de Ancoragem de Plataformas Oceânicas considerando diversos Tramos e o Trecho Enterrado de Linha. (Projeto de Graduação do Curso de Engenharia Civil). Universidade Federal do Rio de Janeiro, 2010.

OLF Norwegian Oil Industry Association - OLF 10 Year Operability Survey of Norwegian FPSOs. Disponível em http://www.norskoljeoggass.no/PageFiles /10605/10\%20year\%20FPSO\%20survey. $\% 20$ Final\%20report.pdf(2011). Acesso em 15/07/2013.

OLIVEIRA, M. F. Metodologia para Aplicação de Fontes Renováveis de Energia Elétrica em Plataformas Marítimas de Produção de Petróleo e Gás Natural. (Tese de Doutorado em Engenharia Elétrica - Departamento de Engenharia de Energia e Automação Elétricas). Escola Politécnica da Universidade de São Paulo, 2013.

PRICE, R.Marine and Offshore Applications: The Coen \& Hamworthy Combustion Handbook: Fundamentals for Power, Marine \& Industrial Applications, v. 8, p. 431. Disponível emhttp://books.google.com.br/books?hl= pt-

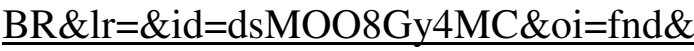
pg=PA431\&dq=FPSO+power+generatio n\&ots=T5oLB8qq1A\&sig=AdtVRIOsQd fbsOBRnBKqoTl8r3Y\#v=onepage \&q $=\mathrm{F}$ PSO\%20power\%20generation \&f $=$ false $(2$ 013). Acesso em 15/06/2013.

RBEPG- Revista Brasil Energia Petróleo e Gás. Artigo "O País dos FPSOs”Edição388pp 36-41, Março, 2013.
TAVARES, C. A. A.O Sistema Elétrico da Plataforma P-43 e suaSimilaridade com o Sistema Elétrico de Potência, 2010 .

TOLMASQUIM, M.T. (Coordenador) Geração de Energia Elétrica no Brasil. Editora CENERGIA-Interciência, Rio de Janeiro (2005)

MENESES, E.L.O Uso de Turbinas a Gás para Geração de Energia Elétrica em Plataformas.(Trabalho de Conclusão de Curso de Graduação em Tecnologia em Construção Naval) Universidade Estadual da Zona Oeste, Rio de Janeiro, 2011.

SIEMENS PRESS RELEASE 1 "Siemens to acquire the Rolls-Royce Energy gas turbine and compressor business and enter into a long-term technology partnership" Disponível emhttp://www.siemens.com/press/pool/d e/pressemitteilungen/2014/corporate/201 4-q2/AXX20140536e.pdf - Acesso em $20 / 12 / 2014$

SIEMENS PRESS RELEASE - 2 "Siemens announces agreement to acquire Dresser-Rand" Disponível emhttp://www.siemens.com/press/pool/d e/pressemitteilungen/2014/corporate/AX X20140967e.pdf - Acesso em 20/12/2014 